\title{
Subdural haematoma mimicking empyema in a patient with multiple sclerosis treated with immunosuppressive monoclonal antibody
}

\author{
Marcin Ciesielka', Anna Jamroz-Wiśniewska², Marcin Bąk' , Paulina Chmielewska', \\ Małgorzata Nowakowska', Konrad Rejdak ${ }^{2}$ \\ 'Student Research Group of Neurology, Medical University, Lublin, Poland \\ ${ }^{2}$ Chair and Department of Neurology, Medical University, Lublin, Poland \\ Ciesielka M, Jamroz-Wiśniewska A, Bąk M, Chmielewska P, Nowakowska M, Rejdak K. Subdural hematoma mimicking empyema in a patient with \\ multiple sclerosis treated with immunosuppressive monoclonal antibody. J Pre-Clin Clin Res. 2018; 12(4): 120-122. doi: 10.26444/jpccr/101603
}

\begin{abstract}
Subdural empyema (SE) and subdural haematoma (SH) can present similar radiological signs on magnetic resonance imaging (MRI) of the brain. Natalizumab, a monoclonal antibody used in the treatment of multiple sclerosis (MS), exerts immunosuppressive effects in the CNS and can rarely be complicated by progressive multifocal leukoencephalopathy. In a 39-year-old female treated because of MS with natalizumab, control MRI showed massive thickening of the meninges and multiple fluid reservoirs, described as most probably empyemas and meningitis. The patient denied head trauma. Laboratory tests did not show inflammation. Orthopantomogram showed dental pathologies - a possible source of infection. Despite intense treatment, the MRI lesions remained unchanged. After neurosurgical intervention, chronic SH was diagnosed. A radiological picture of $\mathrm{SH}$ and $\mathrm{SE}$ could be difficult to differentiate. The aim of the article is to show how unspecific signs in neuroimaging can be caused by chronic $\mathrm{SH}$.
\end{abstract}

\section{Key words}

multiple sclerosis, natalizumab, subdural haematoma, brain empyema

\section{INTRODUCTION}

Subdural haematoma is a severe condition, first described by Rudolf Virchov in 1857, as "an internal haemorrhagic pachymeningitis" [1]. Nowadays, over 150 years later, despite advanced imaging techniques, diagnosis of this condition can be difficult. It is especially difficult to differentiate intracranial subdural empyaema (ISDE) with chronic subdural haematoma (CSDH), because their presentation in neuroimaging can be similar $[2,3]$. The case is presented of CSDH mimicking ISDE in a patient with multiple sclerosis (MS) treated with immunosuppressive monoclonal antibody. The aim of the study is to emphasize how the unspecific signs in neuroimaging can be caused by CSDH.

\section{CASE REPORT}

In November 2016, a 39-year-old female patient was admitted to the Neurological Department for the administration of the next dose of natalizumab. In 2009, the patient was diagnosed with MS and from 2011-2015 she was treated with interferon beta-1b. In October 2015, because of highly-active MS (two relapses during the last year of interferon beta-1b therapy and five new demyelinating lesions on control magnetic resonance imaging, MRI), treatment with natalizumab was initiated. The neurological examination carried out on admission revealed: dysarthria, spastic paresis of left limbs, central paresis of the right VII cranial nerve, and Babinski sign on the left side. Expanded Disability Status Scale (EDSS)

Address for correspondence: Anna Jamroz-Wiśniewska, Chair and Department of Neurology, Medical University, Lublin, Poland

E-mail: annajamrozwisniewska@umlub.pl

Received: 5 November 2018; Acepted: 17 December 2018 score was determined as four points on a scale of $0-10$ in 0.5 increments. Scoring is based on examination by a neurologist.

After one year of natalizumab treatment, a control MRI was performed. Besides the known demyelinating lesions, it showed massive thickening of the meninges and multiple fluid reservoirs (up to $12 \mathrm{~mm}$ ) (Fig. 1,2), which were described
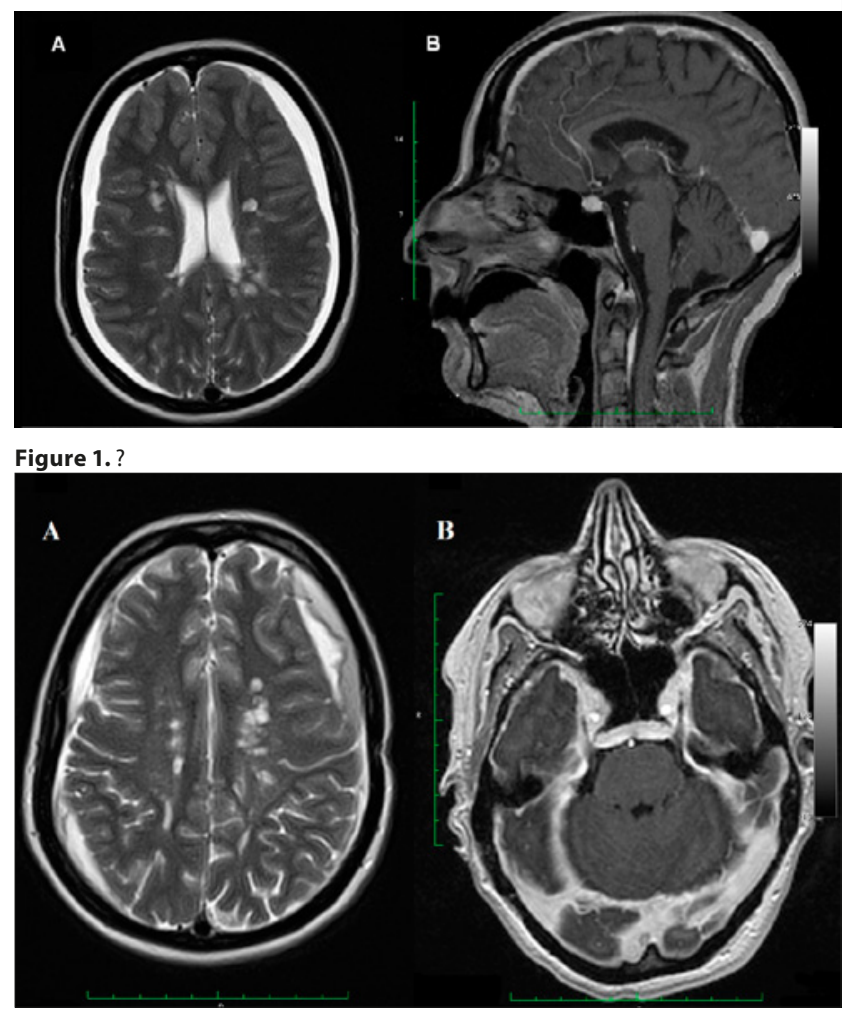

Figure 2. ? 
by a radiologist as most probably empyaema and meningitis. In the beginning, the patient denied having head trauma in the last three months. The patient complained of headache and dizziness associated with sudden head movement. She denied having nausea, fever was absent. Slight nuchal rigidity was present.

Laboratory tests did not show raised inflammatory markers (C-reactive protein: $0.780 \mathrm{mg} / \mathrm{l}$, White Blood Cells: $9.24 / \mu 1$. procalcitonin level $-<0.01 \mathrm{ng} / \mathrm{ml}$ ). Due to the suspicion of meningitis cerebrospinal fluid, an examination was carried out which revealed a raised level of protein $(112 \mathrm{mg} / \mathrm{dl})$, without any other indications of meningitis. Samples of cerebrospinal fluid, urine and blood were taken for microbiological investigation. Cultures were negative. Latex agglutination assay for Neisseria meningitidis, Haemophilus influenza B, and Streptococcus pneumoniae was performed, but the results were negative.

Orthopantomogram showed dental pathologies, which could be the possible source of neuroinfection. Subdural empyaema (SE) was still considered, even without elevation of inflammatory markers, because the patient was treated with natalizumab which is a monoclonal antibody that has potent immune suppressive activity. It prevents adhesion of leukocytes to endothelial cells and their penetration to the CNS, this is why it increases the risk of neuroinfection. Because of the suspicion of SE, empiric broad-spectrum intravenous antibiotic therapy was applied (metronidazol, vancomycin, cefamandol).

Despite dental treatment and empiric antibiotic therapy that lasted over one month, control MRI results remained unchanged. After another neurosurgical consultation, trepanation was carried out to finally determine the nature of changes on the MRI. Aspirated fluid contained blood. This fact led to the diagnosis of CSDH and allowed discharge of the patient after the procedure. Later, she recalled that about two months earlier she had had a mild head trauma due to hitting her head against a closet in the kitchen.

\section{DISCUSSION}

Chronic subdural haematoma is a collection of blood between the inner layer of the dura mater and the arachnoid mater [4]. There is no universally accepted formal definition when subdural haematoma becomes "chronic" [5]. The pathophysiology of CSDH is not fully understood [6]. Annual incidence of CSDH is about 1-5.3 cases per 100,000 population with an increasing incidence, and generally occurs in the elderly, with a male dominance $[4,6]$. It is frequently diagnosed in emergency departments and treated in neurosurgery departments. It is also it is one of the most frequent types of intracranial haemorrhage [6-7]. CSDH are usually located on the most curved frontal or occipital convexity [4].

Symptoms of CSDH include headache, seizures, decreased memory, cognitive impairment, speech disorders, confusion, difficulty in swallowing, gait impairment, weakness or numbness of limbs and face, drowsiness, behaviour disorders, coma, balance problems, dysesthesia, and rarely urinary incontinence or alternation of vision $[1,4,6-7]$. CSDH can also occur without symptoms. CSDH is usually associated with a history of mild head trauma, an injury which may go unnoticed - half of the patients with $\mathrm{CSDH}$ do not remember a head injury or report it as only minor [4]. Lack of trauma in anamnesis and unspecific changes in imaging complicate diagnosis of CSDH. In the presented case, initially the patient denied previous head trauma, only after surgery she confirmed having had a mild trauma. She had forgotten about it, especially as there was no loss of consciousness, bruising, vomiting, or even nausea.

What is important, is that $20 \%$ of patients with $\mathrm{CSDH}$ have some non-vascular neurological diseases [6]. Risk factors include long-term heavy alcohol use, nonsteroidal anti-inflammatory drugs or anticoagulants, intracranial hypotension, implantation of vascular shunts [4, 6-7]. The patient in this case was not taking any drugs increasing the risk of haemorrhage, and did not have any other evident risk factors of intracranial haemorrhage.

There is lack of uniformity in the treatment of CSDH. In the literature there exist various treatment strategies. Surgical techniques are concentrated on decompression of cerebral hemisphere and preventing recurrence $[1,8]$.

$\mathrm{CSDH}$ is usually diagnosed by a computed tomography (CT) scan. It is usually hypodense, but isodensity can also be observed [4]. It is really important to remember that even acute SDH can appear isodense on CT scan [9]. CSDH may also cause a displacement of ventricular system and midline shift [7]. Diagnosis of a subdural fluid collection on CT is challenging, subdural haematoma, subdural empyaema, dural metastases with haemorrhagic pachymeningitis should always be considered [10]. Lymphoma, sarcoma, ischemic stroke, infectious and autoimmune issues can also mimic subdural haematoma $[1,11]$.

Magnetic resonance imaging (MRI) could be helpful in diagnosis - it is more sensitive than CT in determining size and internal structures of CSDH [1]. CSDH is usually slightly hypointense to isointense relative to gray matter on short TR/TE images (TR - repetition time; TE - echo time) [12]. Gadolinium enhanced MRI reveals enhancing and thickened dura sometimes with enhancing membranes. It is also very helpful in differential diagnosis with dural metastases, in contrast to $\mathrm{CSDH}$ in such cases MRI reveals intense and homogenous enhancement [10].

$\mathrm{SE}$ is a loculated suppuration located similarly to $\mathrm{CSDH}$ between the dura mater and the arachnoid. SE is rare, but is a serious infection with a high mortality rate, which varies from 9\% - $48 \%$ [13-14]. Cranial SE occurs more often in children and young adults, with a male dominance [10, 13]. Cranial SE is usually located over the convexity. Most cranial subdural empyaemas are sterile (17.6\%), and the most common organism are Streptococcus Milleri (17.3\%) and Staphylococcus haemolyticus (7.3\%) [13]. Microbiological investigation often does not provide an answer; therefore, in the presented case, negative cultures did not completely exclude empyaema.

Symptoms of SE include high fever, seizures, meningism, disturbances of consciousness, localizing signs, symptoms of increased intracranial pressure (headache, nausea, vomiting). The onset of symptoms is commonly rapid [14]. Clinical presentation is non-specific, and has many common symptoms with CSDH. Cranial SE is usually a complication of laryngological infections (sinusitis or otitis), but it can stem from mastoiditis, infected cranial procedures, dental complications, meningitis, previous head trauma, and bacteriemic seeding of a previous subdural haematoma [3, $10,13-14]$. Conditions or diseases diminishing the patient's 
resistance to infection may also lead to SE development [13].

There are non-specific changes in laboratory analysis: WBC - white blood cells, CRP - C-reactive protein and ESR - erythrocyte sedimentation rate may be elevated, and in the CSF. The gold standard in diagnosis is MRI with gadolinium enhancement which reveals lesions with higher sensitivity than CT. Diffusion-weighted imaging (DWI) MRI can discriminate SE from other fluid collections [10].

Natalizumab, used in the treatment of relapsingremitting MS, is an inhibitor of a 4 subunit of the $\alpha 4 \beta 1$ integrin, preventing it from binding to vascular cell adhesion molecule 1 (VCAM-1). This results in decreased immune surveillance within the CNS. The most frequent serious adverse event is infection (1.9\%) [15]. As natalizumab exerts immunosuppressive effects in the CNS, therapy with this drug can rarely be complicated by progressive multifocal leukoencephalopathy (PML) due to reactivation of the John Cunningham (JC) virus [16]. Although the patient has now been treated with natalizumab for almost three years, in her case, it did not cause any kind of neuroinfection. Her neurological state and EDSS (Expanded Disability Status Scale) score have been stable. However, due to the mechanism of action of the drug with accompanying inflammatory dental abnormalities in the patient, on the radiological description of MRI, intracranial subdural empyaema (ISDE) was suspected. It was finally excluded after neurosurgical intervention.

\section{CONCLUSIONS}

The presented case shows the difficulties in the differential diagnosis between CSDH and ISDE. They can have many similar signs; therefore, ISDE always requires a high clinical suspicion, especially in patients on immunosuppressive treatment. Clinical presentation and radiological findings are essential for the proper diagnosis are; sometimes, however, neurosurgical intervention is necessary.

\section{REFERENCES}

1.Illescu IA. Current diagnosis and treatment of chronic subdural haematomas. J Med Life. 2015; 8(3): 278-284.

2. French H, Schaefer N, Keijzers G, Barison D, Olson S. Intracranial subdural empyema: a 10-year case series. Ochsner J. 2014; 14(2): 188-94.

3. Doan N, Patel M, Nguyen HS, Mountoure A, Shabani S, Gelsomino M, et al. Intracranial subdural empyema mimicking a recurrent chronic subdural hematoma. J Surg Case Rep. 2016; 9: 1-2.

4. Yadav YR, Parihar V, Namdev H, Bajaj J. Chronic subdural hematoma. Asian J Neurosurg. 2016; 11(4): 330-342.

5. Iorio-Morin C, Touchette C, Lévesque M, Effendi K, Fortin D, Mathieu D. Chronic Subdural Hematoma: Toward a New Management Paradigm for an Increasingly Complex Paradigm. J Neurotrauma 2018; 35(16): 1882-1885.

6. Neto JF, Araujo JLV, Ferraz VR, Haddad L, Veiga JCE. Chronic subdural hematoma: epidemiological and prognostic analysis of 176 cases. Rev Col Bras Cir. 2015; 42(5): 283-287.

7.Gelabert-Gonzalez M, Rico-Cotelo M, Aran-Echabe E. Chronic subdural hematoma. Med Clin (Barc). 2015; 144(11): 514-519.

8. Liu W, Bakker NA, Groen RJM. Chronic subdural hematoma: a systematic review and meta-analysis of surgical procedures. J Neurosurg. 2014; 121(3): 665-673.

9. Grelat M, Madkouri R, Bousquet O. Acute isodense subdural hematoma on computed tomography scan - diagnostic and therapeutic trap: a case report. J Med Case Rep. 2016; 10: 43.

10. De Beer MH, Van Gils P, Koppen H. Mimics of subacute subdural hematoma in the ED. Am J Emerg Med. 2013; 31(3): 634.e1-3.

11. Catana D, Koziarz A, Cenic A, Siddharth N, Singh S, Almenawer SA, et al. Subdural Hematoma Mimickers: A Systematic Review. World Neurosurg. 2016; 93: 73-80.

12. Fobben ES, Grossman RI, Atlas SW, Hackney DB, Goldberg HI, Zimmerman RA, et al. MR Characteristics of Sudural Hematomas and Hygromas AT 1.5T. AJR Am J Roentgenol. 1989; 153(3): 589-595.

13. De Bonis P, Anile C, Pompucci A, Labonia M, Lucatntoni C, Mangiola A. Cranial and spinal subdural empyema. Br J Neurosurg. 2009; 23(3): 335-340.

14. Jaskólski DJ. Ropniaki wewnątrzczaszkowe. Aktualn Neurol 2014; 14(2): 112-116.

15. Butzkueven H, Kappos L, Pellegrini F, Trojano M, Wiendl H. Patel RN, et al. Efficacy and safety of natalizumab in multiple sclerosis: interim observational programme results. J Neurol Neurosurg Psychiatry 2014; 85(11): 1190-1197.

16. Natalizumab. In: Aronson JK, editors. Meyler's Side Effects of Drugs. $16^{\text {th }}$ ed. Elsevier; 2016. p. 33-36. 